forces increase in the KTG; flexion, extension, internal and external rotation muscle forces increased in the GPTG ( $p<0,05$ for all). But there was no significant difference between the two groups.

Table 1. The level of pain in GPTG and KTG

\begin{tabular}{|c|c|c|c|c|c|c|}
\hline & \multicolumn{3}{|c|}{ GPTG $($ mean \pm sd) } & \multicolumn{3}{|c|}{$\mathrm{KTG}($ mean $\pm \mathrm{sd})$} \\
\hline & $\begin{array}{l}\text { Before } \\
\text { Therapy }\end{array}$ & $\begin{array}{c}\text { After } \\
\text { Therapy }\end{array}$ & $p$ & $\begin{array}{l}\text { Before } \\
\text { Therapy }\end{array}$ & $\begin{array}{c}\text { After } \\
\text { Therapy }\end{array}$ & $\mathrm{p}$ \\
\hline Night pain severity & $6,69 \pm 3,4$ & $3,62 \pm 2,9$ & $<0,001$ & $5,28 \pm 3,18$ & $2,32 \pm 2,23$ & $<0,001$ \\
\hline Rest pain severity & $3,97 \pm 3,31$ & $2,45 \pm 2,78$ & 0,005 & $2,96 \pm 3,29$ & $1,08 \pm 1,75$ & 0,031 \\
\hline Pain severity with motion & $8,07 \pm 1,81$ & $5,14 \pm 2,47$ & $<0,001$ & $7,72 \pm 2,03$ & $4,36 \pm 2,21$ & $<0,001$ \\
\hline Severity of general pain & $7,76 \pm 4,54$ & $4,66 \pm 3,99$ & $<0,001$ & $8,40 \pm 3,13$ & $4,8 \pm 2,69$ & 0,005 \\
\hline
\end{tabular}

Active internal and external rotation ROM increased in KTG $(p<0,05)$. Functionality and disability scores were improved in two groups. It was detected that while there was no difference between the groups in terms of the values of muscle strength, functionality and disability scores.

Conclusions: The kinesiologic band can be used as an supportive therapy method in the early shoulder treatment program because of it provides painless shoulder motion to clinicians. We consider that KT applications in addition to general physical therapy applications may have positive effects in the treatment of impingement syndrome.

Disclosure of Interest: None declared

DOI: 10.1136/annrheumdis-2017-eular.4613

\section{THU0723-HPR A PHYSIOTHERAPY-LED IN-PATIENT INTENSIVE REHABILITATION PROGRAMME FOR ANKYLOSING SPONDYLITIS: FOLLOW-UP OUTCOMES}

C. Clarke $^{1}$, P. Taggart ${ }^{1}$, J. Monaghan ${ }^{1}$, J. McKnight ${ }^{2}$, A. Cairns ${ }^{2}$.

${ }^{1}$ Physiotherapy; ${ }^{2}$ Rheumatology, Musgrave Park Hospital, Belfast, United Kingdom

Background: Physiotherapy and exercise are highly important in the management of Ankylosing Spondylitis (AS). Physiotherapy is delivered to patients with AS in either in-patient or out-patient settings. Knowledge of the effectiveness of an in-patient delivered programme is useful for physiotherapists in assisting patients to achieve their goals.

Objectives: To assess the short-term effectiveness of an intensive rehabilitation programme using BASMI and EASI-QOL outcomes, and long-term patient satisfaction and physical activity behaviour and adherence to exercise plan.

Methods: Thirty-two AS patients (25 males and 7 females) admitted to an inpatient rheumatology ward underwent a 1 to 2 -week physiotherapy-led intensive rehabilitation programme and were then discharged with a home exercise programme. Pre/post rehabilitation BASMI scores were available for 26 patients. The primary outcome measure was the proportion of patients achieving an improvement on BASMI scores at discharge. Secondary outcome measures included improvements in physical activity levels and adherence to home exercise plan for longer than 3 months which was obtained via a postal patient satisfaction and physical activity questionnaire achieving a response rate of $50 \%(n=16)$.

Results: Improvements in BASMI scores was achieved in $69 \%$ of patients $(n=18)$ at the end of the in-patient rehabilitation period. Improvements in EASI-QOL were achieved in $83 \%$ of patients $(n=15)$ at the end of the in-patient rehabilitation period. Ninety- four percent of patients $(n=15)$ increased their physical activity levels after discharge, with $81 \%(n=13)$ of patients maintaining their home exercise programme for 3 months or more. Thirty-seven percent $(n=7)$ of patients carry out at least 150 minutes of physical activity per week (National Recommended Physical Activity Guidelines is 150 minutes/week of moderate intensity).

Conclusions: This recent audit shows the effectiveness of an intensive physiotherapy-led in-patient rehabilitation programme for Ankylosing Spondylitis improving BASMI scores in the short-term and increasing physical activity behaviour over the long-term. Future work will aim to compare demographics and medical treatment differences between improvers and non-improvers.

Disclosure of Interest: None declared

DOI: 10.1136/annrheumdis-2017-eular.3799

\section{THU0724-HPR SELF-MANAGEMENT EXERCISE PROGRAM ASSOCIATED TO SPA THERAPY INCREASED THE PHYSICAL ACTIVITY LEVEL OF PEOPLE WITH SYMPTOMATIC KNEE OSTEOARTHRITIS: A QUASI-RANDOMIZED CONTROLLED TRIAL}

C. Gay ${ }^{1}$, C. Auclair ${ }^{2}$, N. Boisseau ${ }^{3}$, L. Gerbaud ${ }^{4}$, E. Coudeyre ${ }^{1} .{ }^{1}$ Physical and Rehabilitation Medicine Department, Clermont-Ferrand University Hospital, Clermont Auvergne University, INRA, Human Nutrition Unit UMR1019, Clermont Ferrand; ${ }^{2}$ Public Health Department, Clermont-Ferrand University Hospital, Clermont Auvergne University, PEPRADE, EA 4681; ${ }^{2}$ Laboratory of the Metabolic Adaptations to Exercise under Physiological and Pathological condition (AME2P), Clermont Auvergne University, EA 3533, Clermont-Ferrand; ${ }^{4}$ Public Health Department, Clermont-Ferrand University Hospital, Clermont Auvergne University, PEPRADE, EA 4681, Clermont Ferrand, France

Background: Treating knee osteoarthritis $(\mathrm{OA})$ in the medical phase is today well standardized. Guideline orientated approaches aiming at increasing physical activity (PA), improving pain and disability.

Objectives: To assess effectiveness of self-management exercise program associated to spa therapy at 3 month on the improvement of physical activity (PA) level, disability, pain, anxiety, fears and believes in symptomatic knee osteoarthritis people.

Methods: Prospective, multicentric, quasi-randomized controlled trial with alternate month design method (one month periods). People with symptomatic knee OA people (stage I-IV, Kelgren and Lawrence scale) with low and moderate PA level were included in 3 spa therapy resorts. Intervention group (IG) received 5 self-management exercise sessions ( $1 \mathrm{~h} 30$; education, aerobic, strength training, range of motion) + information booklet +18 sessions (1h) of conventional spa therapy (STC). Control group (CG) received information booklet +18 sessions of STC. The primary outcome was changes at 3 months in PA level (IPAQ short form score) and secondary outcomes were WOMAC function, pain (VAS), HAD anxiety/depression, KOFBeQ fears and believes changes.

Results: 131 subjects were included. The mean age was 65.6 years $[ \pm 6.7]$. WOMAC function score was 22.1/68 [ \pm 11.3$]$ and pain was 4.6/10 [ \pm 1.9$]$ at inclusion. Both groups significantly increased PA level measured with continuous IPAQ total score (MET-minutes/weeks), with superiority for IG $(+77.8 \% ; p=0.0062)$ than CG $(+50.7 \% ; p=0.0099)$. There was no change in setting time. Disability $(-11.3 \% ; p=0.0370)$ and pain $(-15.2 \% ; p=0.0032)$ also decreased significantly for both groups. Anxiety $(-11.6 \% ; p=0.0195)$ and fears and believes $(-18.2 \%$; $\mathrm{p}=0.0146$ ) decreased significantly only in intervention group. Other data will be presented later.

Conclusions: This study confirms the impact of STC on disability and pain and gives news data's on physical activity level. Self-management exercise program improve anxiety, fears and believes. Complex educational strategies comprising information booklet with or without self-management exercise program can be proposed and adapted to OA phenotypes.

References:

[1] Fernandes, Linda, Kåre B. Hagen, Johannes W. J. Bijlsma, Oyvor Andreassen, Pia Christensen, Philip G. Conaghan, Michael Doherty, et al. EULAR Recommendations for the Non-Pharmacological Core Management of Hip and Knee Osteoarthritis. Annals of the Rheumatic Diseases 72, no. 7 (July 2013): 1125-35.

[2] Gay, C., A. Chabaud, E. Guilley, and E. Coudeyre. Educating Patients about the Benefits of Physical Activity and Exercise for Their Hip and Knee Osteoarthritis. Systematic Literature Review. Annals of Physical and Rehabilitation Medicine 59, no. 3 (June 2016): 174-83

Disclosure of Interest: None declared

DOI: 10.1136/annrheumdis-2017-eular.1929

\section{THU0725-HPR COST EFFECTIVENESS ANALYSIS OF ABATACEPT COMPARED WITH TNF INHIBITORS IN PATIENTS WHO ARE POSITIVE FOR ANTI-CITRULLINATED PROTEIN ANTIBODIES BASED ON RESULTS FROM AN OBSERVATIONAL TRIAL}

S. Johal ${ }^{1}$, E. Alemao ${ }^{2}$. ${ }^{1}$ PAREXEL International, London, United Kingdom; ${ }^{2}$ Bristol-Myers Squibb, Princeton, United States

Background: Anti-citrullinated protein antibodies (ACPA) are highly specific to RA and patients (pts) who are ACPA positive (+) tend to develop more severe, erosive disease than ACPA-negative pts. ${ }^{1}$ In an observational study exploring the status of ACPA on RA treatment response to abatacept (ABA) or a TNF inhibitor (TNFi), mean (SE) changes from baseline in CDAl at 6 months was -8.8 for ABA and -5.6 for TNFi initiators. ${ }^{2}$

Objectives: To evaluate the costs and benefits of treating pts with RA who are ACPA+ with ABA vs TNFi on background MTX.

Methods: An economic analysis was carried out estimating lifetime direct costs and quality-adjusted life years (QALYs) of ACPA+ pts with RA treated with ABA or TNFi from a UK National Health Service (NHS) perspective. QALYs are a measure of disease burden, adjusted to reflect quality of life lived. As data for the economic analysis were derived from a real-world study, an "average" pt was modelled, whose baseline characteristics were based on the observational study. CDAl changes at 6 months for each treatment were converted to HAQ changes, and disease progression was based on HAQ score changes over a lifetime. Continuation of therapy was based on rates from the real-world study. Mean long-term survival on treatment with $A B A$ or TNFi was derived from the literature. $^{3}$ In the base case, pts discontinuing ABA or TNFi moved to palliative care (MTX). Direct medical costs and quality of life scores were correlated to HAQ scores. ${ }^{3,4}$ Costs included hospitalizations, joint replacements and treatment costs. Estimates of differences in costs and QALYs between ABA and TNFi initiators were used to calculate an incremental cost-effectiveness ratio (ICER; cost per QALY gained). The annual cost of TNFi was calculated as an average of TNFi drugs in the UK ( $£$ 9113). For ABA, an average cost of five biologics was used ( $£$ 9244) to reflect a realistic cost to NHS UK. A sensitivity analysis examined the effect of varying the input parameters of efficacy, cost and utilities on costs and outcomes.

Results: Based on an "average" pt from the observational study, the total estimated QALYs for ABA and TNFi initiators were 6.4 and 6.27, respectively. Total lifetime costs were $£ 41,378$ and $£ 40,627$, respectively. The lifetime cost for 